\title{
Unit Density
}

National Cancer Institute

\section{Source}

National Cancer Institute. Unit Density. NCI Thesaurus. Code C105705.

The number of arbitrary units of something contained in a specific volume. 